\title{
Does MRI Acoustic Noise Affect Chemosensory Perception?
}

\author{
Remco C. Havermans ${ }^{1,2}$ (D) Anouk E. M. Hendriks ${ }^{1}$
}

Received: 12 October 2018 / Accepted: 6 January 2019/Published online: 25 January 2019

(C) The Author(s) 2019

\begin{abstract}
Introduction Sounds can affect food and flavor perception. For example, loud noise can affect taste perception. In the present study, we examined whether exposure to $\sim 80$-dB magnetic resonance imaging (MRI) acoustic noise affects taste perception.

Methods Participants $(N=27)$ came to the lab twice for a taste test, a smell test, and evaluating sweet and savory food items: once when continuously being exposed to loud MRI acoustic noise and once when being exposed to much quieter background noise. It was hypothesized that exposure to loud noise would impair taste perception, especially identifying and detecting sweet taste, and that it would decrease liking for the taste of particularly sweet foods.

Results Neither overall taste detection nor sweet taste detection was affected by exposure to MRI acoustic noise. Further, we found no effect of noise on sweet food liking, but exploratory analyses do imply such an effect of MRI noise on liking for savory foods, with these foods being significantly less liked when exposed to MRI acoustic noise.

Conclusion We conclude that loud noises do not necessarily affect chemosensory perception.

Implications MRI acoustic noise specifically does not seem to have a relevant effect on smell and taste.
\end{abstract}

Keywords Chemosensory perception $\cdot$ Food liking $\cdot \mathrm{fMRI} \cdot$ Noise $\cdot$ Smell $\cdot$ Taste

\section{Introduction}

Sounds affect the way food is experienced. For example, Zampini and Spence (2004) investigated if the perceived crispness of potato chips is modulated by the sound of the biting action. Participants ate potato chips in a soundattenuated booth in front of a microphone. Both the overall sound level as the higher frequencies were manipulated (i.e., amplified at a pre-specified level) and fed back to the participant via a pair of headphones. Amplifying either the overall sound level or just the higher frequencies significantly increased the perceived crispness and freshness of the potato chips (see also Vickers 1983, 1984).

Woods et al. (2011) investigated whether background noise can impact the perceived taste, mouthfeel, and evaluation of

Remco C. Havermans

r.havermans@maastrichtuniversity.nl

1 Centre for Healthy eating and Food Innovation, Laboratory of Behavioural Gastronomy, Maastricht University Campus Venlo, Venlo, The Netherlands

2 Faculty of Psychology and Neuroscience, Clinical Psychological Science, Maastricht University, Maastricht, The Netherlands food. The researchers found that exposure to loud white noise (75-85 dB), but not quiet white noise (approximately $50 \mathrm{~dB}$ ), led their participants rating sweetness and saltiness of various sweet and savory food items as less intense (experiment 1). This effect might be ascribed to the loud background noise distracting the participants; but in a second experiment, employing the same white noise conditions, the researchers found that loud white noise increased perceived crunchiness across various food items. In both experiments, the loud noise also seemed to negatively affect food liking. The latter finding is in line with research by Seo and Hummel (2011) who found in one of their experiments that the experienced pleasantness of a noise (e.g., jazz drum or a crying baby) affected the liking for various odors.

It appears that background noise can affect both smell and taste perception. Indeed, Stafford et al. (2012) found that participants were impaired in discriminating alcohol levels of alcoholic drinks varying in alcoholic content when exposed to $80 \mathrm{~dB}$ of "hardcore" club music and a simultaneous shadow task in which the participants had to listen to news information and repeat it. Furthermore, the $80-\mathrm{dB}$ background music alone also appeared to affect sweetness ratings. In another study, these researchers found the same effect of the same distractive music and task at sound pressure levels (SPLs) of either 80 or $100 \mathrm{~dB}$ (Stafford et al. 2013). 
Stafford et al. (2013) prefer to explain their findings in terms of distraction. However, more recent research by Yan and Dando (2015) suggest that the effect of background noise on taste perception reflects a very different mechanism. They exposed their participants once to airline cabin noise (at approximately $85 \mathrm{~dB}$ ) and once to near silence. In both conditions, participants rated the perceived intensity of different concentrations of a sweet, sour, salty, bitter, and an umami solution. For all concentrations, sweet taste was perceived as less intense when exposed to loud airline cabin noise. Conversely, at higher concentrations, umami taste was perceived as slightly more intense when exposed to loud noise. This gustatory effect of loud noise could not be ascribed to being distracted, as the simulated airline cabin noise did not affect participants' performance on a simple reaction time task. Yan and Dando speculate that the apparent effect of particularly loud noise on taste perception reflects mechanical stimulation of the chorda tympani, a cranial nerve that innervates two thirds of the tongue and transfers taste signals from the taste buds to the solitary tract in the brain stem. The chorda tympani nerve bundle crosses the tympanic membrane in the middle ear, close to the eardrum. If true, one would then expect an effect of any loud enough noise on taste perception.

In sum, loud background noise seems to affect taste perception and for any researcher this means that $\mathrm{s} / \mathrm{he}$ has to carefully control for or consider ambient sound levels when collecting data regarding taste perception. This recommendation is straightforward enough, but may pose a considerable challenge when examining the neurobiology of flavor processing. Studies charting the neurophysiological correlates of taste perception in human subjects mostly involve functional magnetic resonance imaging (fMRI). Typically, participants are placed in an MRI scanner and exposed to specific liquid tastants (see e.g., Dalenberg et al. 2015) or certain liquid foods (e.g., Frost et al. 2015). But MRI, particularly echo planar imaging (as in an fMRI study), is notoriously noisy.

MRI acoustic noise is due to Lorentz forces generated by gradient current switching causing the gradient coils to vibrate in their mountings. These Lorentz forces are proportional to magnetic field strength, and hence, MRI acoustic noise is generally louder with stronger magnetic fields. A 3-Tesla (T) MRI scanner can produce acoustic noise with SPLs as high as $130 \mathrm{~dB}$ (Price et al. 2001; Ravicz et al. 2000). Hearing protection in the form of earplugs and protective earmuffs combined is estimated to reduce SPL with no more than $40 \mathrm{~dB}$ (Ravicz, et al.). It thus seems that the SPL of acoustic noise experienced in a 3-T MRI scanner is high enough to affect taste perception.

In the present study, we examined whether specifically the noise of an MRI scanner can affect the sense of taste. Moreover, we explored whether this noise might also affect smell perception. In light of the findings by Woods et al. (2011) and Yan and Dando (2015), we hypothesized that: (1) taste function, particularly identifying and detecting sweet taste, is impaired when exposed to $\sim 80$-dB MRI acoustic noise; and (2) liking for specifically sweet food is decreased when having to consume sweet food items while being exposed to $\sim 80$-dB MRI acoustic noise. ${ }^{1}$

\section{Method}

The present study was preregistered on April 10, 2018 at the Open Science Framework (https://osf.io/knexa/).

\section{Participants}

In light of the findings by Yan and Dando (2015), who reported a "considerable decrement" (p. 593) in ratings of sweet taste, we planned to recruit a sample of participants large enough to detect a medium effect of noise on taste; specifically that it would negatively impact taste, particularly sweet taste identification and detection. On the basis of a power analysis in $\mathrm{G}^{*}$ Power (Faul et al. 2007) for a paired samples $t$ test, employing an alpha rejection criterion of 5 and $80 \%$ statistical power (1 - beta) for the detection of a medium effect size, we recruited and tested 27 participants. Of these participants, apart from experimental measurements, we only documented their age and gender (seven men, 20 women; $M$ age = 27.7 years, $S D$ age $=8.7$ years).

Participants were recruited by posted advertisements at Maastricht University Campus Venlo. They had to be between 18 and 50 years old and have a (self-reported) normally functioning sense of hearing, smell, and taste. Participants were excluded from the study if they reported having tinnitus, having food allergies, regularly smoking cigarettes (more than 5 cigarettes per day), being pregnant, or using medication at a frequency and dose impairing one's sense of taste or smell. The study protocol was evaluated and approved by a local institutional review board (i.e., the Maastricht University Ethical Research Committee of Psychology and Neuroscience, reference number ERCPN-186_05_12_2017).

\section{Materials}

Audio-Related Materials In both conditions, participants wore open-back stereo headphones (SAMSON RH100; SAMSON Technologies, Hicksville NY, USA) connected to a receiver (Teufel KB42 CD receiver with USB input; Teufel, Berlin, Germany). Participants were thus exposed to MRI acoustic noise.

\footnotetext{
${ }^{1}$ We initially planned to test a third hypothesis, predicting that subjectively rated intensity of specifically sweet foods is decreased when having to listen to loud MRI acoustic noise. We eventually felt testing this hypothesis would add little to the other two hypotheses and thus decided to refrain from assessing self-reported taste intensity.
} 
Using the music creation software GarageBand (Apple, Cupertino CA, USA), we created a 40-min long digital audio file comprising noise of mostly functional imaging sequences recorded from a 3-T Siemens Magnetom Allegra head-only scanner. These recordings included localizer, MDEFT imaging, MP RAGE, and functional TR2 $(64 \times 64$ voxel matrix/ $128 \times 128$ voxel matrix) with (or without) delay imaging. The different scanning noises were presented in a random order with each noise repeated three times before switching to the next MRI sequencing noise. Between each block of three repetitions, there was a brief $(\sim 1 \mathrm{~s})$ interval. All MRI sequencing noises were presented against continual $60-\mathrm{dB}$ background noise comprising a looped recording of the liquid helium coolant pump. The complete series of MRI acoustic noise was 20min long, and this whole series was repeated once to create a recording with a total duration of $40 \mathrm{~min}$. We estimated that an experimental session would not exceed $20 \mathrm{~min}$, but wanted to have a recording longer than that, just in case. The original audio WAV files (including the recording of the coolant pump) used in the current study are publicly shared on the Open Science Framework (https://osf.io/sdexw/).

Taste Strips Taste Strips (Burghart Messtechnik, Wedel, Germany) is a commercially available test to determine an individual's gustatory function (see Landis et al. 2009). The test comprises 16 paper strips impregnated with one of four concentrations of the basic tastants (i.e., sweet [sucrose], sour [citric acid], salty [sodium chloride], and bitter [quinine hydrochloride] taste). The strips were presented in one of two pseudo-randomized sequences (which sequence was determined randomly for each individual participant), with each strip being placed in the center of the anterior extended tongue. Participants were instructed to close their mouth and to move the strip around for whole-mouth testing. They were then asked to indicate with each strip whether they could taste anything and if so whether it tasted sweet, sour, salty, or bitter (forced choice). In between trials, participants rinsed their mouth with tap water. Completion of the test (approximately $8 \mathrm{~min}$ ) results in a total maximum score of 16 correct taste detections ( $\max 4$ for each basic taste quality).

Sniffin' Sticks The smell test comprised the commercially available 12-item odor identification Sniffin' Sticks test (Burghart Messtechnik, Wedel, Germany). It is a brief test that has been studied extensively and has been shown to differentiate between normal sense of smell and hyposmia (Hummel et al. 2001).

The Sniffin' Sticks are felt tipped pens filled with an odorous solution. The cap is removed from the pen, and its tip is held $2 \mathrm{~cm}$ under the nose in front of the nostrils of the participant for about $3 \mathrm{~s}$. The participant then has to indicate the odor $\mathrm{s} /$ he believes to have just smelled from a 4-option choice card. Participants thus try to identify 12 different but common smells (e.g., orange, cinnamon, leather, fish). In the current study, the pens were presented in a random order for each participant in each of the two sessions. The score on this brief $\sim 3$-min test is the total number of correctly identified odors with a maximum of 12 .

Line Scales The participants rated their wanting of various food items on separate 100-mm line scales ranging from "not at all" ( 0 ; outer left anchor) to "very much" (100; outer right anchor). Similarly, participants rated their momentary liking for the various food items on 100-mm line scales, also ranging from "not at all" (0; outer left anchor) to "very much" (100; outer right anchor). Further, just before sampling the various food items, the participants rated their momentary hunger and thirst on separate 100-mm line scales, ranging from "not at all" ( 0 ; outer left anchor) to "extremely" (100; outer right anchor). An example of these line scales is shared on the Open Science Framework (https://osf.io/3hyfv/).

Foods Participants received various food items to consume and evaluate. They consumed two savory and two sweet items. These items included: cheese (Albert Heijn, plakken Goudse Kaas, $390 \mathrm{kcal} / 100 \mathrm{~g}$ ) on a cracker (Carr's Table Water Cracker, $412 \mathrm{kcal} / 100 \mathrm{~g}$ ); a 20-ml aliquot of tomato juice (Albert Heijn, $22 \mathrm{kcal} / 100 \mathrm{ml}$; savory drink) served in a transparent disposable 40-ml cup; a single white grape ( $75 \mathrm{kcal} / 100 \mathrm{~g}$ ); a $20-\mathrm{ml}$ aliquot of apple juice (Albert Heijn, $47 \mathrm{kcal} / 100 \mathrm{ml}$ ) served in a transparent disposable $40-\mathrm{ml}$ cup.

\section{Procedure and Design}

Participants took part in a within-subjects study, meaning they were tested once when exposed to MRI acoustic noise (condition noise) and once in the absence of such noise (condition control). To control for level of anxiousness experienced during the noise condition, we first primed the participant briefly to the MRI acoustic noise (at $\sim 60 \mathrm{~dB}$ ) and in the noise condition told him/her that s/he would be exposed to a maximum SPL of $\sim 80 \mathrm{~dB}$; whereas in the control condition, we stated that $\mathrm{s} /$ he might be exposed to $\sim 80 \mathrm{~dB}$ MRI acoustic noise at any randomly determined interval during the test session. The order of taking part in the conditions was counterbalanced between participants. Participants were always tested individually, and all sessions were scheduled in the morning (between 9 am and noon).

Upon arrival for the first session, the participant was led to the laboratory (a temperature regulated, pressurized, $\sim 15-\mathrm{m}^{2}$ room) and informed of the general aim and procedure of the experiment. Informed consent was obtained from all individual participants included in the study. After providing informed consent, the experimenter would position the headphones and briefly prime the participant with the MRI acoustic noise (as described above). 
Subsequently, the participant would receive the Sniffin' Sticks followed by the Taste Strips test. Depending on the session, the participant would listen to either $\sim 80$-dB MRI scanning acoustic noise, or $\sim 60-\mathrm{dB}$ background noise while undergoing these psychophysical tests. After a brief pause, during which the participant was allowed to momentarily take off the headphones and was asked to rate his/her momentary hunger and thirst on separate line scales while the experimenter fetched the test food items from the adjacent laboratory kitchen, the session was resumed with the participant having to consume and rate the cracker with cheese, the $20-\mathrm{ml}$ serving of tomato juice, the grape, and the 20-ml apple juice. These items were consumed in a random order while, again, having to listen to loud MRI acoustic noise or the much quieter sound of the coolant pump (depending on the condition). For each item, the participant was first asked to look at it and rate his/her wanting to consume the item on a $100-\mathrm{mm}$ line scale, and then to consume the item and rate liking for its flavor on a separate $100-\mathrm{mm}$ line scale. This concluded the session.

The second experimental session was planned for the next week under the provision of at least a 6-day interval (i.e., washout period) between the first and second sessions (the longest interval between sessions was 16 days). The second session was identical to the first session with the exception that participants now had to listen to 80-dB MRI noise if they listened to $60-\mathrm{dB}$ background noise in the previous session and vice versa. Upon conclusion of the second session, the participants were asked to indicate what they thought the experiment was about after which they were debriefed. None of the participants guessed any of the specific hypotheses as stated above in the "Introduction," though many expressed their awareness of the wider aim to examine the effect of MRI noise on taste perception (of which they also were informed prior to taking part in the study). Upon completion of both test sessions, the participants received a $15 €$ gift card for remuneration and (when applicable) were compensated for travel expenses.

\section{Data Handling and Analysis Plan}

To test the two aforementioned hypotheses, we planned to conduct one sided paired samples $t$ tests comparing condition noise with condition control for (1) sweet taste identification scores (ranging between 0 and 4), (2) overall taste identification score (ranging between 0 and 16), and (3) liking ratings for sweet food items (apple juice and grape). Note that the latter outcome variable is a mean score, averaging the liking for the apple juice and for the grape.

As pre-registered, we conducted further analyses, exploring with two-sided paired samples $t$ tests any differences between conditions (noise versus control) concerning: odor identification, salty taste identification, sour taste identification, bitter taste identification, averaged wanting and liking ratings for the savory food items, and averaged wanting for the sweet food items.

For all the planned tests, we first visually inspected data for possibly influential outliers (by plotting the data for each DV as histograms) and checked for normality of the outcome variable with the Shapiro-Wilks test. In case of non-normality, we conducted and report the nonparametric Wilcoxon signed rank test instead of the initially planned paired samples $t$ test.

All analyses were conducted in R (R Core Team 2018) by using RStudio (RStudio Team 2018). The anonymized raw data (scanned into two PDF files, 1 for the noise condition and 1 for the control condition) are available on the Open Science Framework. The Excel file worksheets we created and used for data analyses and plots, along with the R scripts, are publicly shared on the Open Science Framework too (https://osf.io/uymtw/).

\section{Results}

\section{Taste and Odor Identification}

Figures 1 and 2 display box plots for the Taste Strips test and the 12-item Sniffin' Sticks odor identification test for each within-subject condition (control versus noise), respectively. A paired samples $t$ test comparing within-subjects conditions noise and control concerning the overall score on the Taste Strips test revealed no significant difference in taste function between the conditions $\left(M_{\text {control }}=12.48, S D_{\text {control }}=2.33\right.$, $M_{\text {noise }}=12.44, S D_{\text {noise }}=3.03 ; t[26 d f]=0.08, p=.47$ ).

A Wilcoxon signed rank test comparing noise and control concerning specifically the sweet taste score of the Taste Strips test also revealed no significant difference between conditions $\left(M_{\text {control }}=3.59, S D_{\text {control }}=.54, M_{\text {noise }}=3.52\right.$, $\left.S D_{\text {noise }}=.94 ; V=38.5 p=.31\right)$.

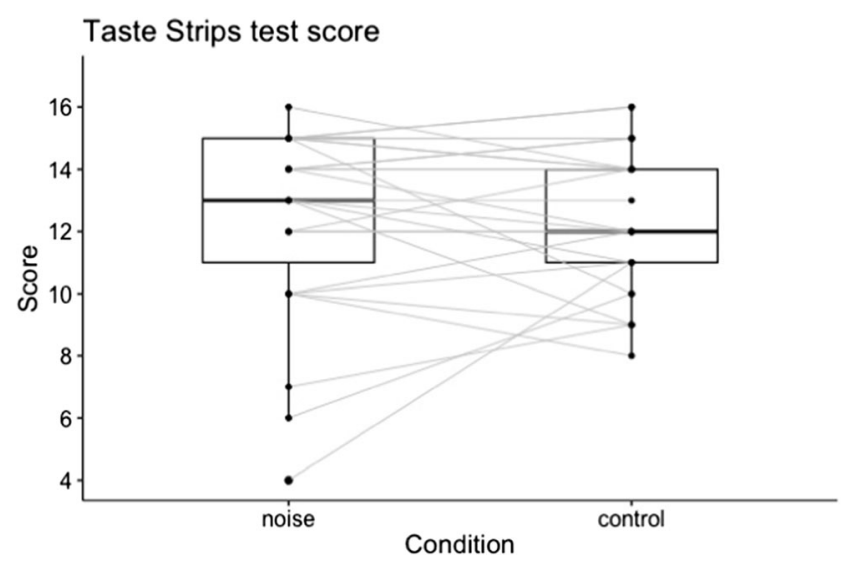

Fig. 1 Box plots of the Taste Strips scores for condition noise versus control. The lines indicate the paired scores of individual participants 
Sniffin' Sticks test score

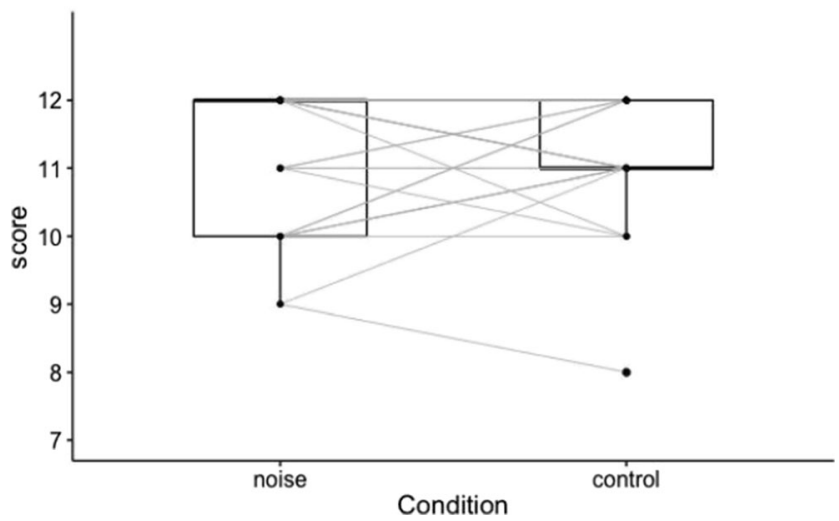

Fig. 2 Box plots of the Sniffin' Sticks scores for condition noise versus control. The lines indicate the paired scores of individual participants

To explore the possibility of MRI acoustic noise not having a meaningful effect on taste function, we conducted two one-sided $t$ tests for equivalence using the TOSTER R package (see Lakens 2017). We set symmetric equality bounds based on a medium effect size (i.e., Cohen's $d_{\mathrm{z}}=.35$ ), arguing that if the observed difference falls within these bounds, this difference is hardly relevant. This medium effect corresponds to an 0.8 point difference in total Taste Strips test score between the two conditions. The $t$ tests for both the lower and upper bound proved statistically significant $(p s<.05)$ and thus the observed difference in Taste Strips scores falls within the specified equality bounds.

We conducted a two-sided Wilcoxon signed rank test exploring the difference in odor identification scores between conditions control $\left(M_{\text {control }}=11.26, S D_{\text {control }}=0.94\right)$ and Noise $\left(M_{\text {noise }}=11.15, S D_{\text {noise }}=1.03\right)$. No statistically significant difference was found, $V=70.5, p=.55$.

\section{Sweet Food Liking and Wanting}

Figure 3 displays the mean liking and wanting ratings ( \pm SEM) for the sweet foods (i.e., apple juice and grape). We conducted a one sided paired samples $t$ test comparing the conditions control and noise on the averaged liking for the consumed sweet foods, and found no significant difference $\left(M_{\text {control }}=67.50, S D_{\text {control }}=13.73\right.$, $\left.M_{\text {noise }}=64.35, S D_{\text {noise }}=12.95 ; t[26 d f]=1.18, p=.12\right)$. Again, to explore the possibility of the MRI noise not having a relevant effect on sweet food liking, we conducted two one-sided $t$ tests for equivalence using the TOSTER package for R (Lakens 2017). We set symmetric equality bounds based on a medium effect size (Cohen's $d_{\mathrm{z}}=.35$ ) and argue that if the observed difference in sweet food liking would fall within these bounds, this could hardly be called relevant. A medium effect
Sweet food ratings

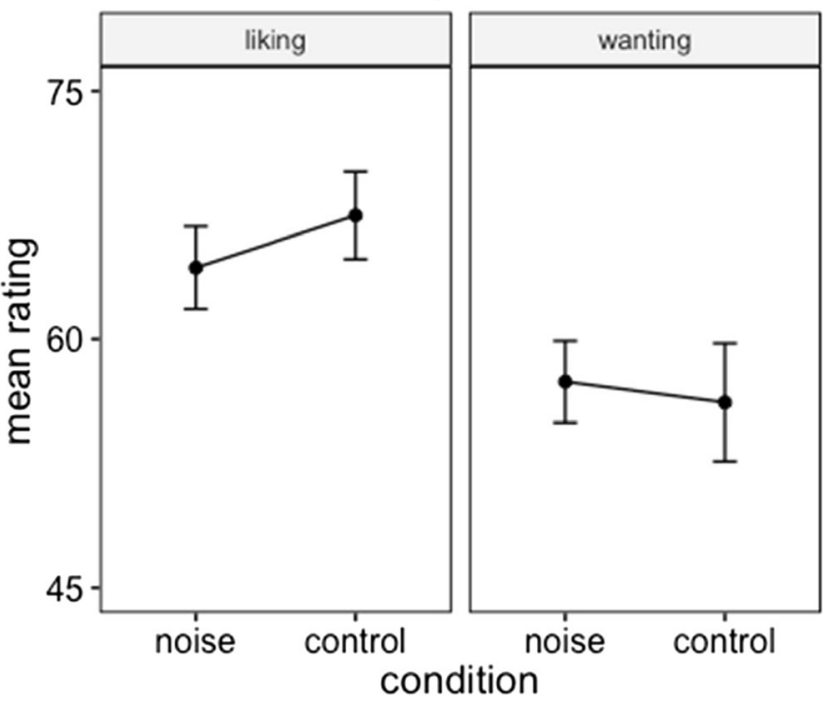

Fig. 3 Mean liking (left panel) and wanting (right panel) ratings ( \pm SEM) for the sweet food items in condition noise versus control

would correspond to a little less than a 5 -mm difference on the $100-\mathrm{mm}$ line scale. The $t$ test for the upper bound proved statistically significant $(p<.05)$, but not for the lower bound $(p>.05)$, implying that the failure to find a significant decrease in liking for sweet foods may reflect a type II error.

We further explored wanting ratings for the sweet food items and found no significant difference between control $\left(M_{\text {control }}=56.19, S D_{\text {control }}=18.49\right)$ and noise $\left(M_{\text {noise }}=57.43\right.$, $\left.S D_{\text {noise }}=12.79\right), t(26 d f)=-.32 p=.75$.

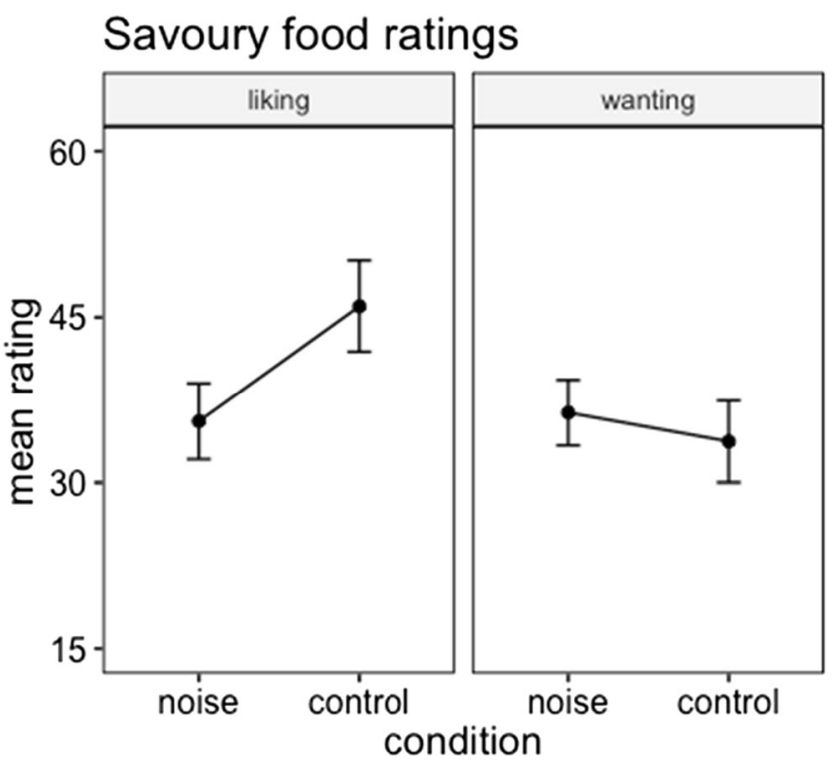

Fig. 4 Mean liking (left panel) and wanting (right panel) ratings ( \pm SEM) for the savory foods in condition noise versus control 


\section{Savory Food Liking and Wanting}

Figure4 displays the mean liking and wanting ratings for the savory foods (i.e., tomato juice and cheese on cracker) for within-subject conditions noise versus control. Exploratory $t$ tests were conducted for liking and wanting ratings for the savory foods. Regarding liking for savory food, there was a significant difference between conditions $\left(M_{\text {control }}=46.00, S D_{\text {control }}=21.40 ; M_{\text {noise }}=35.56\right.$, $\left.S D_{\text {noise }}=17.91\right), t(26 d f)=2.60, p=.02$, implying that savory foods were liked less when having to listen to loud MRI acoustic noise.

No significant difference in wanting the savory food was found when comparing conditions control $\left(M_{\text {control }}=33.72\right.$, $\left.S D_{\text {control }}=19.28\right)$ and noise $\left(M_{\text {noise }}=36.22, S D_{\text {noise }}=15.47\right)$, $t(26 d f)=-.75, p=.46$.

\section{Discussion}

In the present study, we examined whether exposure to MRI acoustic noise affects chemosensory perception. Neither taste nor odor identification was significantly affected. More specifically, on the basis of previous findings by Woods et al. (2011) and Yan and Dando (2015), we hypothesized that sweet taste identification would be negatively affected by MRI acoustic noise, but we found no support for that either.

We also failed to observe a clear effect of loud noise on sweet food liking, but as outlined above in the "Results" section, this may reflect a type II error. Indeed, we did find a significant effect of loud MRI noise on savory food liking, that is, savory foods were significantly less liked when exposed to MRI acoustic noise. This finding is in line with previous results reported by Woods et al. (2011) who indicated that food was seemingly liked less when exposed to loud white noise and who also found that noise diminished perceived food sweetness and saltiness. It is possible that the participants in our study also found the savory food items a little less savory (i.e., salty) when exposed to MRI acoustic noise. This then would have diminished their liking for the savory food items.

Another possible explanation for our results is that participants disliked the MRI acoustic noise and this transferred to the evaluation of the various foods. In the study by Woods et al. (2011), food liking correlated with the degree to which the participants found the loud white noise annoying. Food was less liked, the more the noise was disliked. We did not assess the degree to which our participants found the MRI acoustic noise annoying, but the loud knocking, tapping, thumping, and chirping noise is generally regarded as a profound source of discomfort in patients having to undergo an MRI scan (Alibek et al. 2014; Morton and Gildersleve 2000). It is safe to assume that the MRI acoustic noise we exposed our participants to was in fact annoying and the ensuing discomfort of that experience tainted food evaluation, leading to an observed tendency for decreased food enjoyment (irrespective of its taste quality). Indeed, this is very similar to what Seo and Hummel (2011) found in their research: the valence of a noise presented before and during an odor readily transferred to the odor. We agree this is post hoc speculation based on exploratory analyses. Nonetheless, we feel it is a tentative explanation that warrants further research. It should be noted that this presumed effect does not imply that participants necessarily came to dislike the foods we served them when exposed to acoustic noise. They merely seemed to like food slightly less when exposed to loud and (presumably) annoying MRI acoustic noise. We should state that this latter finding is in direct contrast with a study by Ferber and Cabanac (1987) who found that loud 90-dB background noise (or music) increased liking for a sweet taste solution at specific concentrations. Although this discrepancy is somewhat puzzling, the merit of the study by Ferber and Cabanac is moot, considering that with just 10 participants it is severely underpowered, and the pairwise analyses (at least 30) were left uncorrected for multiple testing.

Importantly, MRI acoustic noise, while certainly annoying, did not seem to exert an effect on chemosensory perception. However, we exposed our participants to all noises comprising a typical MRI scanning session and not just echoplanar imaging noises associated with an fMRI scan. Indeed, from the standpoint of a neuroscientist interested in examining the neurological underpinnings of taste and smell, the only relevant noise in this respect is the sound of functional MRI sequencing. In this respect, it should also be noted that the use of $\sim 80$-dB SPLs is at the lower end of noise level when considering MRI acoustic noise. Exposure to much higher SPLs (e.g., $90 \mathrm{~dB}$ ) however was deemed unethical as prolonged exposure to such loud noise may inflict hearing damage. More importantly, it was also thought to be unnecessary, as significant effects of auditory noise on taste perception has been reported for noise levels around $80 \mathrm{~dB}$ (see e.g., Woods et al. 2011).

One might argue that the awkwardness of participating in an fMRI taste or food perception study-lying in a confined space, being fed liquid foods or taste solutions through a plastic mouthpiece-may still distort taste perception. Any testing situation in experimental psychology (including cognitive neuroscience studies) is unusual, but that fact alone does not necessarily invalidate its findings (see Mitchell 2012). Nonetheless, there is research that indicates that a supine position weakens olfactory perception (Lundström et al. 2006) and according to the law of inverse effectiveness, stating that multisensory integration is enhanced when the best unisensory response is 
(relatively) weak, the optimal condition for smelling (i.e., sitting upright) in the present study might have biased against an effect of the acoustic noise on flavor perception. But the law of inverse effectiveness does not always apply (Holmes 2007) and more importantly, it does not explain the lack of an acoustic noise effect in the current research relative to positive findings of just such an effect in prior research. MRI acoustic noise then does not appear to affect chemosensory perception, but to definitively rule out interference of such noise when studying taste perception in a neuroimaging setting requires a test for such interference with the participants ideally undergoing an actual fMRI scanning session.

The findings from the present study are thus somewhat puzzling as they do not readily elucidate the effects of acoustic noise on taste processing. However, the contention that loud noise affects taste processing because of high SPLs mechanically stimulating the chorda tympani seems false. If that is the case, we should have found a significant effect of MRI acoustic noise on taste perception, but we did not find that. Note though that Woods et al. (2011) and Yan and Dando (2015) did not directly assess psychophysical taste function. They merely asked their participants to indicate subjectively perceived taste intensity on a line scale. Furthermore, it is possible that only exposure to a more continual high SPL with white noise or airline cabin noise (as opposed to the interrupted rhythmic variations of knocking, chirping, and tapping that characterizes MRI acoustic noise) will notably affect taste perception. This possibility too requires further research.

To recapitulate, we found that (f)MRI acoustic noise itself, disregarding potential interactions with other fMRI contextual elements (e.g., heightened arousal, a supine position), does not influence identification of suprathreshold taste and odor stimuli. Further research is needed to examine if fMRI acoustic noise can still systematically confound chemosensory perception within the context of an actual fMRI scanning experiment. Other interesting research questions are to what degree highly annoying sounds (any sound and irrespective of SPL) can negatively affect food liking and whether only loud continual acoustic noise (as opposed to intermittent noise) influences taste perception.

Acknowledgements The Laboratory of Behavioural Gastronomy is supported by the Dutch Province of Limburg. The authors would further like to thank Linda Romanovska for sharing the MRI audio recordings and Ilse Knoops for her aid in recruiting and testing participants.

\section{Compliance with Ethical Standards}

Conflict of Interest $\mathrm{RCH}$ declares that he has no conflict of interest. AEMH declares that she has no conflict of interest.
Ethical Approval All procedures performed in studies involving human participants were in accordance with the ethical standards of the institutional and/or national research committee and with the 1964 Helsinki declaration and its later amendments or comparable ethical standards.

Informed Consent Informed consent was obtained from all individual participants included in the study.

Open Access This article is distributed under the terms of the Creative Commons Attribution 4.0 International License (http:// creativecommons.org/licenses/by/4.0/), which permits unrestricted use, distribution, and reproduction in any medium, provided you give appropriate credit to the original author(s) and the source, provide a link to the Creative Commons license, and indicate if changes were made.

Publisher's Note Springer Nature remains neutral with regard to jurisdictional claims in published maps and institutional affiliations.

\section{References}

Alibek S, Vogel M, Sun W, Winkler D, Baker CA, Burke M, Gloger H (2014) Acoustic noise reduction in MRI using Silent Scan: an initial experience. Diagn Interv Radiol 20(4):360-363. https://doi.org/10. 5152/dir.2014.13458

Dalenberg JR, Hoogeveen HR, Renken RJ, Langers DRM, ter Horst GJ (2015) Functional specialization of the male insula during taste perception. NeuroImage 119:210-220. https://doi.org/10.1016/j. neuroimage.2015.06.062

Faul F, Erdfelder E, Lang A, Buchner A (2007) G*Power 3: a flexible statistical power analysis program for the social, behavioral, and biomedical sciences. Behav Res Methods 39(2):175-191

Ferber C, Cabanac M (1987) Influence of noise on gustatory affective ratings and preference for sweet or salt. Appetite 8(3):229-235. https://doi.org/10.1016/0195-6663(87)90022-5

Frost R, Quiñones I, Veldhuizen M, Alava JI, Small D, Carreiras M (2015) What can the brain teach us about winemaking? An fMRI study of alcohol level preferences. PLoS One 10(3):1-11. https:// doi.org/10.1371/journal.pone.0119220

Holmes NP (2007) The law of inverse effectiveness in neurons and behaviour: multisensory integration versus normal variability. Neuropsychologia 45(14):3340-3345. https://doi.org/10.1016/j. neuropsychologia.2007.05.025

Hummel T, Konnerth CG, Rosenheim K, Kobal G (2001) Screening of olfactory function using a 4-minute odor identification test: reliability, normative data, and investigations in patients with olfactory loss. Ann Otol Rhinol Laryngol 110:976-981. https://doi.org/10.1177/ 000348940111001015

Lakens D (2017) Equivalence tests: a practical primer for t-tests, correlations, and meta-analyses. Soc Psychol Personal Sci 8(4):355-362. https://doi.org/10.1177/1948550617697177

Landis BN, Welge-Luessen A, Brämerson A, Bende M, Mueller CA, Nordin S, Hummel T (2009) "Taste Strips"—a rapid, lateralized, gustatory bedside identification test based on impregnated filter papers. J Neurol 256(2):242-248. https://doi.org/10.1007/s00415009-0088-y

Lundström JN, Boyle JA, Jones-Gotman M (2006) Sit up and smell the roses better: olfactory sensitivity to phenyl ethyl alcohol is dependent on body position. Chem Senses 31(3):249-252. https://doi.org/ 10.1093/chemse/bij025

Mitchell G (2012) Revisiting truth or triviality: the external validity of research in the psychological laboratory. Perspect Psychol Sci 7(2): 109-117. https://doi.org/10.1177/1745691611432343 
Morton G, Gildersleve C (2000) Noise in the MRI scanner. Anaesthesia 55(12):1213-1219. https://doi.org/10.1046/j.1365-2044.2000. 01798-11.x

Price DL, De Wilde JP, Papadaki AM, Curran JS, Kitney RI (2001) Investigation of acoustic noise on $15 \mathrm{MRI}$ scanners from $0.2 \mathrm{~T}$ to 3 T. J Magn Reson Imaging 13:288-293

R Core Team (2018) R: A language and environment for statistical computing [computer software manual]. Vienna, Austria. Retrieved from https://www.R-project.org/

RStudio Team (2018) RStudio: integrated development for R [computer software manual]. Boston, MA. Retrieved from http://www.rstudio. com/

Ravicz ME, Melcher JR, Kiang NY-S (2000) Acoustic noise during functional magnetic resonance imaging. J Acoust Soc Am 108(4):16831696. https://doi.org/10.1121/1.1310190

Seo HS, Hummel T (2011) Auditory-olfactory integration: congruent or pleasant sounds amplify odor pleasantness. Chem Senses 36(3): 301-309. https://doi.org/10.1093/chemse/bjq129
Stafford LD, Fernandes M, Agobiani E (2012) Effects of noise and distraction on alcohol perception. Food Qual Prefer 24:218-224. https://doi.org/10.1016/j.foodqual.2011.10.012

Stafford LD, Agobiani E, Fernandes M (2013) Perception of alcohol strength impaired by low and high volume distraction. Food Qual Prefer 28:470-474. https://doi.org/10.1016/j.foodqual.2012.12.005

Vickers ZM (1983) Pleasantness of food sounds. J Food Sci 48:783-786

Vickers ZM (1984) Crispness and crunchiness-a difference in pitch? J Texture Stud 15:157-163

Woods AT, Poliakoff E, Lloyd DM, Kuenzel J, Hodson R, Gonda H, Batchelor J, Dijksterhuis GB, Thomas A (2011) Effect of background noise on food perception. Food Qual Prefer 22(1):42-47. https://doi.org/10.1016/j.foodqual.2010.07.003

Yan KS, Dando R (2015) A crossmodal role for audition in taste perception. J Exp Psychol Hum Percept Perform 41(3):590-596. https:// doi.org/10.1037/xhp0000044

Zampini M, Spence C (2004) The role of auditory cues in modulating the perceived crispness and staleness of potato chips. J Sens Stud 19(5): 347-363. https://doi.org/10.1111/j.1745-459x.2004.080403.x 